\title{
Testing of Neutron Data for Fe, Cr, Ni based on Integral Experiments
}

\author{
V. N. Koshcheev ${ }^{1, a}$, G. N. Manturov ${ }^{1}$, M. Yu. Semenov ${ }^{1}$, A. M. Tsibouliya ${ }^{1}$ \\ ${ }^{1}$ IPPE, Obninsk, Russia 249033
}

\begin{abstract}
In this work the modern evaluated nuclear data for structural materials such as $\mathrm{Fe}, \mathrm{Cr}$, $\mathrm{Ni}$ are considered, and comparison between the RUSFOND data file library and other libraries is done. Criticality calculations for a set of selected fast uranium and plutonium benchmarks are performed, and calculation results are compared with experimental data. Monte Carlo calculations were performed using continuous energy cross-sections corresponding to different data libraries.
\end{abstract}

\section{Introduction}

National neutron data libraries were recently modified in USA, Europe, Japan, China and Russia. These data libraries are now being tested for further use in applications. The neutron data were mainly tested using criticality calculations for the benchmarks selected by a special procedure from the ICSBEP Handbook. Other tests included 1) calculations of average cross sections on standard and some specially selected spectra, and 2) calculations of the cross sections of neutron removal under the thresholds of detector reactions such as (n,fis)U-238, (n,fis)Np-237, (n,p)Al-27, etc. These results are presented in this work.

\section{Neutron data libraries}

The following neutron data libraries were used: the Russian library ROSFOND presented in 2008 and corrected in 2010 [1]; the Japanese library JENDL-4.0 presented in 2011 [2]; the US library ENDF/B-VII.1 presented in 2011 [3]; the European library JEFF-3.1.1 presented in 2009 [4].

Iron, Chrome and Nickel were selected as the materials to be tested in this work, for they are basic components of structural materials in fast breeder reactors.

\section{Benchmarks used}

The Mughabghab evaluation of resonance integrals (the spectrum of $\sim 1 / \mathrm{E}$ ) was used as a reference [5]. The typical LMFBR specter is used as a $\varphi(\mathrm{LMFBR})$. Average cross sections calculated on the

\footnotetext{
a e-mail: abbn@ippe.ru
} 
U-235 and Cf-252 fission spectra as well as removal cross sections from the EXFOR Library were taken as reference values [6].

The input data and corresponding benchmark criticalities for selected benchmarks were taken from ICSBEP Handbook [7].

The benchmark selection was made by the tools of the DICE database [8].

The criteria for selecting benchmark were 1) a relatively high fission rate above $100 \mathrm{keV}, 2$ ) a relatively high absorption rate above $100 \mathrm{keV}$ which is related to absorptions by structural nuclides.

29 models with structural materials influencing mainly on neutron leakage, and 8 models with structural materials influencing mainly on the absorption rate were selected. This allows one to separate two basic effects due to presence of structural materials.

\section{Results}

The comparison of the results on resonance integrals (the spectrum of $\sim 1 / \mathrm{E}$ ) for $\mathrm{Cr}, \mathrm{Fe}$ and $\mathrm{Ni}$ are presented in Table 1.

Table 1. The comparison of the one group cross sections for various library neutron data.

\begin{tabular}{|c|c|c|c|c|c|c|c|c|}
\hline $\mathrm{Cr}$ & capture & total & $\mathrm{Fe}$ & capture & total & $\mathbf{N i}$ & capture & total \\
\hline $\operatorname{EXP} \varphi(1 / E)$ & 1.000 & & $\operatorname{EXP} \varphi(1 / E)$ & 1.000 & & $\operatorname{EXP} \varphi(1 / E)$ & 1.000 & \\
\hline RF2010 & 0.741 & 1.000 & RF2010 & 1.005 & 1.000 & RF2010 & 1.033 & 1.000 \\
\hline E-VII.1 & 0.750 & 0.942 & E-VII.1 & 1.004 & 0.998 & E-VII.1 & 0.981 & 1.001 \\
\hline JEFF-3.1.2 & 0.742 & 1.002 & JEFF-3.1.2 & 1.002 & 0.999 & JEFF-3.1.2 & 1.033 & 1.000 \\
\hline JENDL-4.0 & 0.756 & 0.942 & JENDL-4.0 & 1.004 & 0.998 & JENDL-4.0 & 1.062 & 1.009 \\
\hline$\varphi(L M F B R)$ & & & $\varphi(L M F B R)$ & & & $\varphi(L M F B R)$ & & \\
\hline RF2010 & 1.000 & 1.000 & RF2010 & 1.000 & 1.000 & RF2010 & 1.000 & 1.000 \\
\hline E-VII.1 & 0.900 & 0.949 & E-VII.1 & 0.972 & 0.998 & E-VII.1 & 0.920 & 1.004 \\
\hline JEFF-3.1.2 & 1.006 & 1.006 & JEFF-3.1.2 & 0.940 & 1.004 & JEFF-3.1.2 & 1.000 & 1.000 \\
\hline JENDL-4.0 & 0.888 & 0.949 & JENDL-4.0 & 0.975 & 1.001 & JENDL-4.0 & 1.005 & 1.018 \\
\hline EXP $\varphi(C f 252)$ & & & EXP $\varphi(C f 252)$ & & 1.000 & EXP $\varphi(C f 252)$ & & 1.000 \\
\hline RF2010 & 1.000 & 1.000 & RF2010 & 1.000 & 1.002 & RF2010 & 1.000 & 1.033 \\
\hline E-VII.1 & 0.964 & 0.980 & E-VII.1 & 1.038 & 0.998 & E-VII.1 & 0.970 & 1.033 \\
\hline JEFF-3.1.2 & 0.998 & 1.001 & JEFF-3.1.2 & 0.945 & 1.010 & JEFF-3.1.2 & 1.000 & 1.033 \\
\hline JENDL-4.0 & 0.801 & 0.972 & JENDL-4.0 & 1.083 & 1.010 & JENDL-4.0 & 1.004 & 1.053 \\
\hline
\end{tabular}

The experimental values of the removal cross sections presented in Table 2 were taken from Ref. [11].

Criticality calculations were performed by MCNP5 [9]. The RF2010 and JENDL-4.0 libraries were converted to the ACE format by NJOY [10]. In the case of ENDF/B-VII and JEFF-3.1.1 libraries, the original files in the ACE format were used. 


\section{WONDER-2012}

Table 2. The comparison of the removal cross sections for various library neutron data.

\begin{tabular}{|c|c|c|c|c|c|c|c|c|c|c|c|}
\hline $\mathrm{Cr}$ & $\begin{array}{c}(n, f) \\
\text { Np237 }\end{array}$ & $\begin{array}{l}(n, f) \\
U 238\end{array}$ & $\mid \begin{array}{l}(n, p) \\
A / 27\end{array}$ & $\mathrm{Fe}$ & $\begin{array}{c}(n, f) \\
N p 237\end{array}$ & $\begin{array}{l}(n, f) \\
U 238 \\
\end{array}$ & $\begin{array}{l}(n, p) \\
A / 27\end{array}$ & $\mathrm{Ni}$ & $\begin{array}{c}(n, f) \\
N p 237\end{array}$ & $\begin{array}{l}(n, f) \\
\cup 238\end{array}$ & $\begin{array}{l}(n, p) \\
A / 27\end{array}$ \\
\hline EXP & & & & EXP & 1.000 & 1.000 & 1.000 & $X P$ & 1.000 & 1.000 & 1.000 \\
\hline 20 & 00 & 000 & .000 & RF2 & 0.826 & 0.902 & 1. & RF2 & 1. & 32 & 75 \\
\hline E-VII. & 1.087 & 065 & 1.016 & $E-V$ & 0.837 & 0.919 & 1.058 & $E-I$ & 1.1 & .030 & .091 \\
\hline JEFF-3.1.2 & 0.993 & 0.998 & 1.000 & JEFF-3.1.2 & 0.831 & 0.912 & 1.080 & JEFF-3.1.2 & 1.060 & 0.982 & 1.075 \\
\hline JENDL-4.0 & 1.124 & 077 & $\mid 1.017$ & \begin{tabular}{|l|} 
JENDL-4.0 \\
\end{tabular} & 0.899 & .965 & .098 & JENDL-4.0 & 1.043 & 0.945 & 1.015 \\
\hline
\end{tabular}

The comparison between calculation and benchmark values for $\mathrm{Fe}, \mathrm{Cr}$ and $\mathrm{Ni}$ (with mentioning which type of their properties, reflection or absorption, is the most essential one in each particular case) is presented in Figs. 1-6.

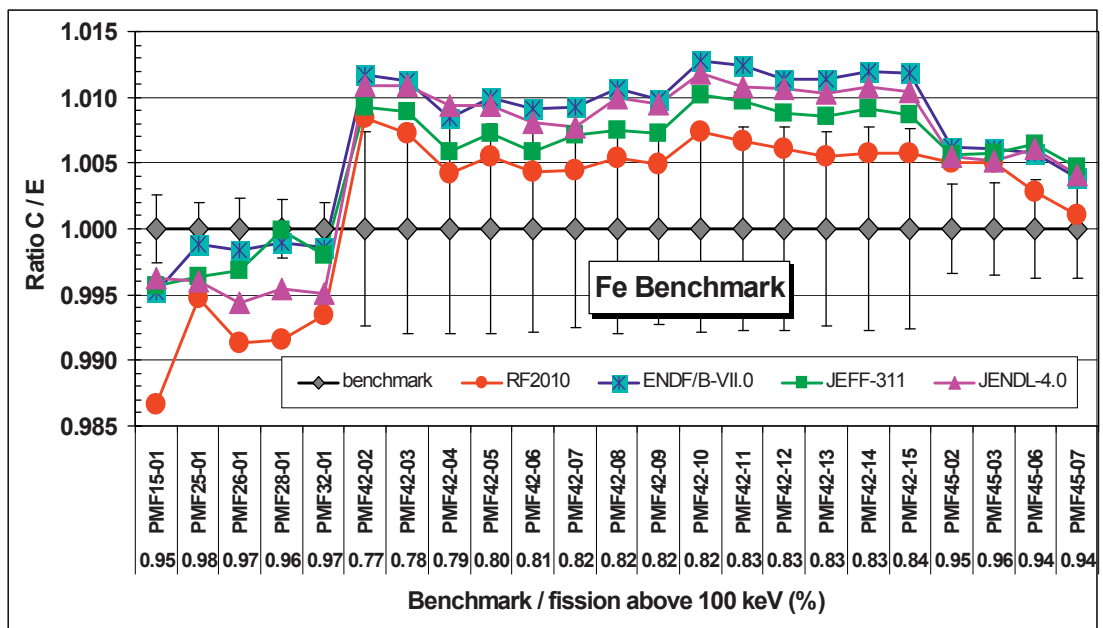

Fig. 1: Fe benchmarks with Pu fuel for various library neutron data

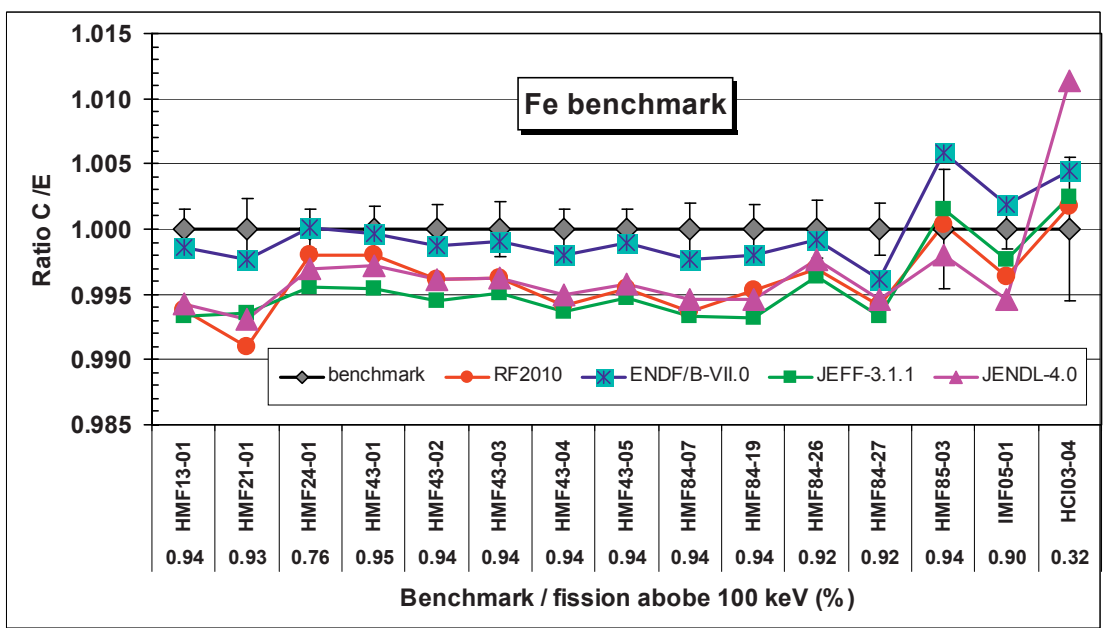

Fig. 2: Fe benchmarks with $U$ fuel for various library neutron data 


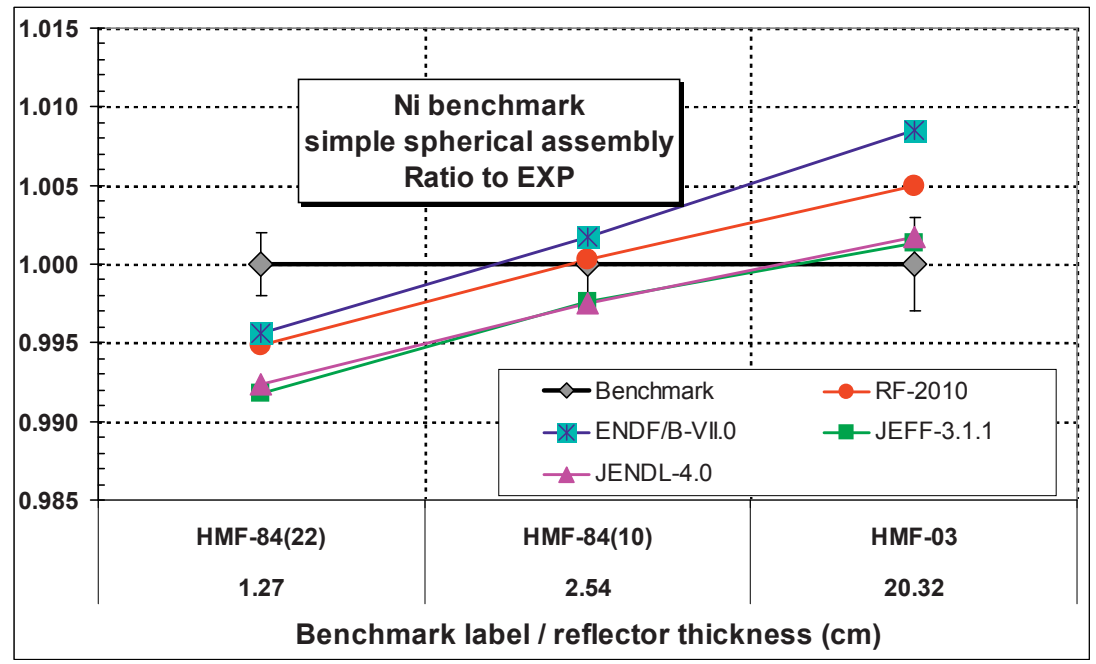

Fig. 3: Ni simple benchmarks for various library neutron data.



Fig. 4: Ni assembly benchmarks for various library neutron data. 


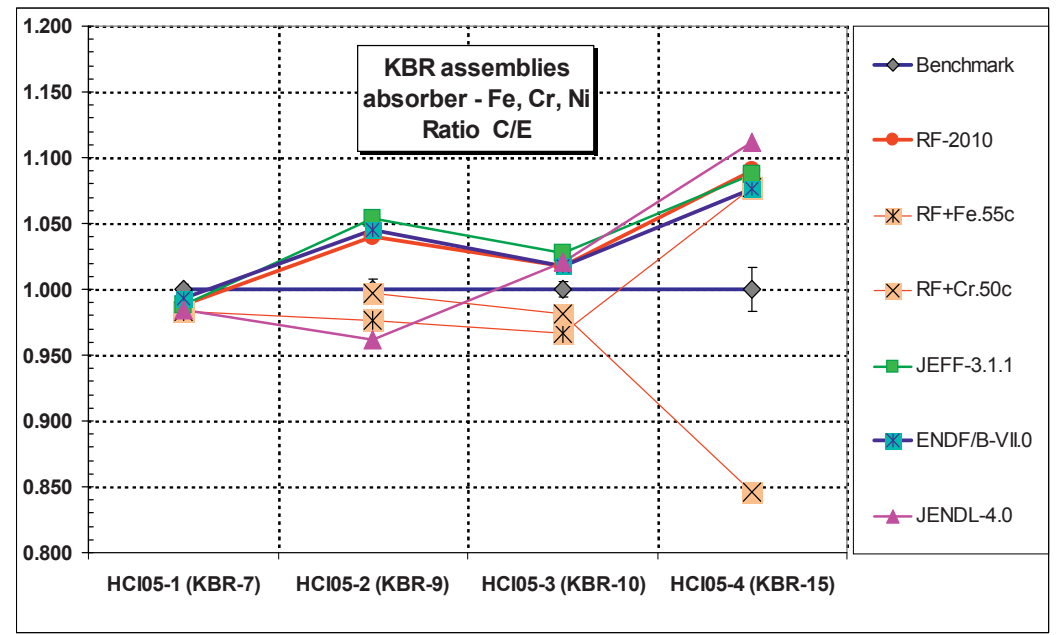

Fig. 5: HCI05 (KBR) benchmarks for various library neutron data.

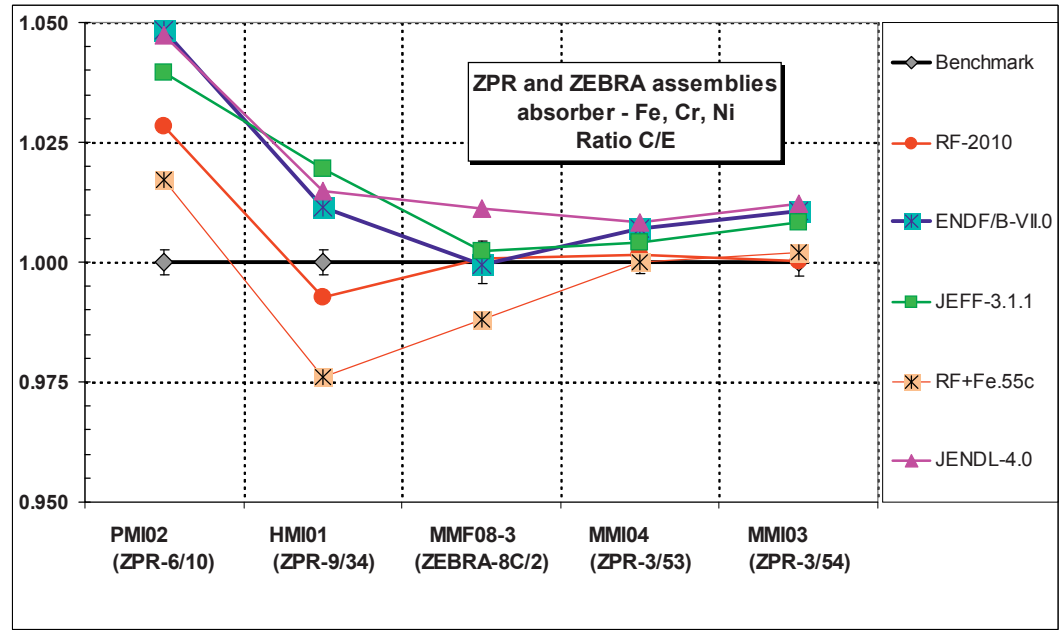

Fig. 6: ZPR and ZEBRA benchmarks for various library neutron data.

\section{Discussion}

According to Table 1, in the last Mughabghab compilation resonance properties were considerably reevaluated for $\mathrm{Cr}$. For other structural nuclides only minor changes took place.

The evaluation of inelastic scattering agrees with experimental data for $\mathrm{Ni}$ only. The removal cross sections of other structural nuclides differ from experiment data (for each type of detector reaction) except for the JENDL-4.0 library.

According to the benchmark values, the reflector properties of Fe are described in the best manner by the ENDF/B-VII.1 library. The results for the PMF042 series contradict with the results for the benchmarks with U fuel.

The calculation results for the Ni-reflected benchmarks reveal some correlation with reflector thickness. Besides, some relationship between calculation values of criticality and neutron specter hardness is observed (see Fig. 3). 
The problem of calculation description of the KBR assemblies (the HCI005 series) is known for a long time [12]. This problem was discussed on the ND2010 conference [13]. The capture process is underestimated in the modern evaluations of $\mathrm{Fe}, \mathrm{Cr}$ and Ni nuclear data as can be seen from Fig. 5. Use of the ENDF/B-V.2 data for $\mathrm{Cr}$ and $\mathrm{Fe}$ (in this evaluation results on integral experiment were taken into account) leads to the opposite effect. So, the truth lies somewhere in between.

\section{Conclusion}

The Fe elastic scattering evaluation by Fu, Perrey et al. (adopted in ENDF/B-VII.1) allowed to describe the Fe-reflected benchmarks from the ICSBEP Handbook in the best way.

Reevaluation of capture cross sections of the $\mathrm{Cr}$ isotopes is desired. It is expected that capture properties should be effectively increased in accordance with the Mughabghab recommendations. To reproduce benchmark values corresponding to the HCI005 series some increase of capture cross sections of $\mathrm{Fe}$ and $\mathrm{Ni}$ (along with $\mathrm{Cr}$ ) is expected.

\section{References}

[1] ROSFOND-2010 Library, Institute of Physics and Power Engineering, Obninsk, Russia (2010). Available online at http://www.ippe.ru/podr/abbn/libr/rosfond.php.

[2] K. Shibata et al., "JENDL-4.0: A new library for nuclear science and engineering," J. Nucl. Sci. Technol.. 48, pp. 1-30, 2011.

[3] M.B. Chadwick et al., "ENDF/B-VII.1 Nuclear data for science and technology: cross sections, covariances, fission product yields and decay data," Nuclear Data Sheets, v.112, 12, 2011

[4] The JEFF-3.1.1 Nuclear Data Library, JEFF Report 22, NEA No. 6807, OECD 2009.

[5] S.F. Mughabghab, "Atlas of Neutron Resonances: Thermal Cross Sections and Resonance Parameters", Elsevier Publisher, Amsterdam, 2006.

[6] Experimental Nuclear Reaction Data (EXFOR / CSISRS),

http://www.nndc.bnl.gov/exfor/exfor00.htm

[7] International Handbook of Evaluated Criticality Safety Benchmark Experiments, OECD/NEA, NEA/NSC/DOC(95)03 (September 2009 Edition)

[8] Ali Nouri, Pierre Nagel, J. Blair Briggs, Tatiana Ivanova. DICE: Database for the International Criticality Safety Benchmark Evaluation Program Handbook, Nucl. Sci. Engin., 145, 2003, p. 1119.

[9] B.B. Forrest, T.E. Booth et al. "MCNP-A General Monte Carlo N-Particle Transport Code, Version 5, Overview and Theory, Volume I", LA-UR-03-1987, LANL, (2003)

[10] R.E. MacFarlane et al. NJOY97.0 Code System for Produsing Pointwise and Multigroup Neutron and Photon Sections from ENDF/B Data. RSIC Peripheral Shielding Routine Collection, PSR-368.

[11] Bethe N.A., Beyster J.R., Carter R.E. Inelastic Cross Sections for Fission Spectrum Neutrons IV, J. Nucl. Energy, 1957, v. 4, p. 147 (EXFOR, subent 11461000).

[12] T.Ivanova, V.Koscheev, M.Nikolaev et al., Calculations of k-infinity of Benchmark Experiments for Compositions of U-235 with Various Structul Matetials. Journal of Nuclear Science and Technology, Proc. of Int. Conf. ND2001, Tsukuba, Japan, 2001

[13] J.Blair Briggs, John D.Bess, Nuclear Data Performance Testing Using Sensitive, but Less Frequently Used ICSBEP Benchmarks. Journal of Korean Physical Society, Vol. 59, №.2, August 2011, pp.1141-1145. 